University of Wollongong

Research Online

Australian Institute for Innovative Materials -

Papers

Australian Institute for Innovative Materials

$1-1-2013$

\title{
Aqueous dispersions of reduced graphene oxide and multi wall carbon nanotubes for enhanced glucose oxidase bioelectrode performance
}

Willo Grosse

University of Wollongong,wmg896@uowmail.edu.au

Joffrey Champavert

University of Wollongong

Sanjeev Gambhir

University of Wollongong, sanjeev@uow.edu.au

Gordon G. Wallace

University of Wollongong, gwallace@uow.edu.au

Simon E. Moulton

University of Wollongong, smoulton@uow.edu.au

Follow this and additional works at: https://ro.uow.edu.au/aiimpapers

Part of the Engineering Commons, and the Physical Sciences and Mathematics Commons

Research Online is the open access institutional repository for the University of Wollongong. For further information contact the UOW Library: research-pubs@uow.edu.au 


\title{
Aqueous dispersions of reduced graphene oxide and multi wall carbon nanotubes for enhanced glucose oxidase bioelectrode performance
}

\begin{abstract}
Aqueous dispersions of reduced graphene oxide ( $\mathrm{rGO}$ ) and multi walled carbon nanotubes (MWCNT) were fabricated through a modified chemical reduction method. The significant advantage of the method developed here is the omission of any stabilising compound or organic solvent to obtain stable rGOMWCNT dispersions. Significantly biological entities, in this case the enzyme glucose oxidase (GOx), can be successfully incorporated into the dispersion. These dispersions were characterised using XPS, SEM, zeta potential and particle size measurements which showed that the dispersion stability is not sacrificed with the addition of GOx, and significantly, the electrical properties of the rGO and MWCNTs are maintained. In this study, rGO acts as an effective dispersing agent for MWCNTs and does not affect the solubility or electroactivity of the GOx. Bioelectrodes fabricated from these rGO-MWCNT-GOX dispersions were characterised electrochemically to test their feasibility in facilitating direct electron transfer (DET) from the redox centre of the enzyme to the electrode. The DET results showed that the specific catalytic current generated at an optimised rGO-MWCNT-GOx electrode was $72 \mathrm{uA} / \mathrm{ug}$ which is 144 times more efficient than other literature values for similar systems. The remarkable specific catalytic current can be attributed to the use of purified enzyme, the efficiency of charge transfer within the rGO-MWCNT composite and the ability of the electrode to facilitate direct electron transfer.
\end{abstract}

\section{Keywords}

aqueous, graphene, performance, oxide, multi, wall, dispersions, carbon, nanotubes, enhanced, reduced, glucose, oxidase, bioelectrode

Disciplines

Engineering | Physical Sciences and Mathematics

\section{Publication Details}

Grosse, W., Champavert, J., Gambhir, S., Wallace, G. G. \& Moulton, S. E. (2013). Aqueous dispersions of reduced graphene oxide and multi wall carbon nanotubes for enhanced glucose oxidase bioelectrode performance. Carbon, 61 (September), 467-475. 


\section{Aqueous Dispersions of Reduced Graphene Oxide and Multi Wall Carbon Nanotubes for Enhanced Glucose Oxidase Bioelectrode Performance}

Willo Grosse, Joffrey Champavert, Sanjeev Gambhir, Gordon G. Wallace* and Simon E. Moulton*

ARC Centre of Excellence for Electromaterials Science, Intelligent Polymer Research Institute, University of Wollongong, New South Wales 2522, Australia.

\section{Abstract:}

Aqueous dispersions of reduced Graphene Oxide (rGO) and multi walled carbon nanotubes (MWCNT) were fabricated through a modified chemical reduction method. The significant advantage of the method developed here is the omission of any stabilizing compound or organic solvent to obtain stable rGO-MWCNT dispersions. Significantly biological entities, in this case the enzyme glucose oxidase (GOx), can be successfully incorporated into the dispersion. These dispersions were characterised using XPS, SEM, zeta potential and particle size measurements which showed that the dispersion stability is not sacrificed with the addition of GOx, and significantly, the electrical properties of the rGO and MWCNTs are maintained. In this study, rGO acts as an effective dispersing agent for MWCNTs and does not affect the solubility or electroactivity of the GOx. Bioelectrodes fabricated from these rGO-MWCNT-GOx dispersions were characterised electrochemically to test their feasibility in facilitating direct electron transfer (DET) from the redox centre of the enzyme to the electrode. The DET results showed that the specific catalytic current generated at an optimized rGO-MWCNT-GOx electrode was $72 \mu \mathrm{A} / \mu \mathrm{g}$ GOx, which is 144 times more efficient than other literature values for similar systems. The remarkable specific catalytic current can be attributed to the use of purified enzyme, the efficiency of charge transfer within the rGO-MWCNT composite and the ability of the electrode to facilitate direct electron transfer.

*Corresponding authors

Phone: +61-2-4221 3127. Fax: +61-2-4221 3114 .

E-mail: smoulton@uow.edu.au; gwallace@uow.edu.au 


\section{Introduction}

Unique electrode architectures have been fabricated from processable solutions of carbon nanotubes. These include fibres [1], films [2] and hydrogels [3], the formation of which usually requires a surfactant or organic solvent [4] to suspend the highly insoluble carbon nanotubes [5]. The addition of surfactants has detrimental effects on the electrical properties of the final electrodes and whilst organic solvents provide an alternative dispersing media to surfactants these have limitations in terms of being inappropriate to use with biological systems [6]. Therefore it would be highly beneficial for carbon nanotubes to be suspended in an aqueous solution, without the need for such surfactants or organic solvents.

Recently, graphene has been praised as a new nanomaterial with the potential for large scale production in aqueous media [7]. Graphene oxide (GO) is a highly dispersible form of graphene due to its surface functionalities, with chemical reagents [8], high temperatures [9] and electrochemical methods widely used to reduce GO into a more conducting form with less basal plane oxygencontaining functionalities [10]. Of significant importance is that the conducting reduced GO (rGO) is stable in an aqueous dispersion and as such has the versatility of being amenable to a range of solution processing techniques.

Graphene oxide-carbon nanotube (GO-CNT) composites have been the subject of significant research interest with studies aimed at characterising the enhanced electrical properties $[11][12][13][14]$ of the composites or modelling the complex interaction between the two allotropes of carbon [15][16][17]. It has been shown that graphene has a higher electroactive surface area than carbon nanotubes [13][18][19]. However, carbon nanotubes can effectively bridge [20] graphene sheets resulting in improvements in sheet resistivity [12] for composite materials. It is still unclear as to how graphene and CNTs interact on a molecular level in solution with some reports suggesting the graphene sheets wrap around carbon nanotubes [18] while others hypothesise that carbon nanotubes 
inhibit the aggregation of suspended graphene sheets [13]. The formation of Schottky barriers play a significant role in determining the electrical properties of the final composite, regardless of what type of graphene or carbon nanotube system is being employed [20][21]. To date the reduction of GOCNT composites has been performed chemically [8], electrochemically [22] or through heat treatment [18][23], and in most cases this reduction has occurred after electrode fabrication or in the presence of stabilising agents such as polyethyleneimine or hydrazine [24].

Carbon nanotubes have been extensively used in electrode structures for enzymatic biosensors [25][26][27] and biofuel cells [28][29][30]. The high aspect ratio and good electrical properties makes them ideal candidates for use with bio-catalysts due to their ability to access the enzyme's active site and facilitate direct electron transfer (DET). Graphene has also received some attention in this regard[31][32]. However, to our knowledge composite graphene-carbon nanotube electrodes have not been investigated for use in biological applications. Essentially, rGO's ability to act as an effective dispersing agent for the MWCNTs and the enzyme in an aqueous system allows the fabrication of novel bioelectrodes.

It is well documented that glucose oxidase physically immobilised in a CNT [33] or rGO [31] electrode can undergo fast electron transfer with the electrode. In the presence of glucose (equation 1) [34], the flavine adenine dinucleotide (FAD) active centre of GOx converts from GOx(FAD) to $\mathrm{GOx}\left(\mathrm{FADH}_{2}\right)$ (equation 2) [35], the oxidised and reduced forms respectively. The electrons generated create a catalytic current that can be measured electrochemically and is one of the ways in which these types of electrodes can be characterised.

$$
\begin{aligned}
& \text { GOx }(\mathrm{FAD})+\text { Glucose } \rightarrow \mathrm{GOx}\left(\mathrm{FADH}_{2}\right)+\text { Glucolactone } \\
& \mathrm{GOx}(\mathrm{FAD})+2 \mathrm{e}^{-}+2 \mathrm{H}^{+} \leftrightarrow \mathrm{GOx}\left(\mathrm{FADH}_{2}\right)
\end{aligned}
$$


Harsh organic solvents have been used to stabilise rGO-CNT suspensions [12], however, these systems are unsuitable for biological applications unless the solvent is completely removed before biological entities are incorporated. It is therefore useful to have a technique that allows for biologically relevant components to be integrated during the solution-processing phase of electrode fabrication. It has been extensively shown that cells [36][37] respond to electrical stimuli and enzymes [32] can be electrically wired to conducting materials, and as such achieving an intimate connection between the biological entity and the materials is critical for the performance of advanced medical devices. The ability to solution process dispersions of conducting materials together with biological entities could facilitate this intimate connection. Additionally, these dispersions allow for existing solution phase fabrication techniques, including fibre fabrication for nerve regeneration [38] and printable implantable electronics [39], which could open the door to a new range of bioelectrodes.

In this paper we report a novel method for the successful formation of aqueous rGO-MWCNT dispersion without the use of other stabilising agents. In addition we show that it is possible to incorporate the enzyme glucose oxidase (GOx) into the dispersion without compromising the biological activity of the enzyme. We show that the electrodes formed from these rGO-MWCNTGOx solutions achieve very efficient DET signals and outperform many of the previously reported rGO and MWCNT based enzyme electrodes in terms of catalytic current. An aqueous based "onepot” processing strategy for the development of biologically compatible carbon electrodes will have a profound effect on the development of advanced electrodes for biomedical applications.

\section{Experimental}

\subsection{Reagents and Materials}

Graphene Oxide (GO) was synthesized from graphite by a modified Hummers method and suspended in MilliQ water [40][41]. Multi-walled carbon nanotubes (MWCNT) (purchased from 
NanoAmor at $99.9 \%$ purity) were used as received without further purification. Hydrazine (SigmaAldrich, 35 wt\% in water) and ammonia solution (Crown Scientific, $28 \mathrm{wt} \%$ in water) were used to chemically reduce GO [7]. Glucose oxidase from Asperilligus niger (GOx) (Sigma-Aldrich) was purified [42] before use and stored at $0.5 \mathrm{mg} / \mathrm{mL}$ in a phosphate buffer solution (PBS) $(\mathrm{pH}=5,50$ $\mathrm{mM})$ at $-80^{\circ} \mathrm{C}$ in small aliquots $(1.5 \mathrm{~mL})$ and was defrosted and stored at $4^{\circ} \mathrm{C}$ when required. The specific activity of the purified GOx was determined using an ABTS Assay [43] and measured to be $\sim 450 \mathrm{U} / \mathrm{mg}$. The purification protocol reported by Gao et al. was slightly modified to ensure sufficient binding of this protein to the anionic exchange column, but otherwise the protocol was adhered to. A $1 \mathrm{mg} / \mathrm{ml}$ solution of poly(ethyleneimine) (SigmaAldrich) was prepared in an aqueous $0.5 \mathrm{M}$ solution of $\mathrm{NaCl}$ (SigmaAldrich). A $1 \mathrm{M}$ solution of D-(+)-glucose in PBS (pH 7.2, $50 \mathrm{mM})$ was prepared and allowed to mutarotate for 24 hours at $4^{\circ} \mathrm{C}$ before use [44]. Glassy Carbon Electrodes (GCE) with a diameter of 3 mm were used for all experiments.

\subsection{Dispersion preparation and characterisation}

MWCNTs were weighed out and added to $10 \mathrm{~mL}$ of GO $(0.5 \mathrm{mg} / \mathrm{mL})$ in water with the following weight percent of MWCNTs; $0 \%, 10 \%, 33 \%, 50 \%$, $85 \%$, $90 \%$ and $100 \%$. In order to suspend the MWCNTs effectively the GO-MWCNT solution was subjected to horn sonication (Branson Digital Sonifier - 500W) for 1 hour (30\% amplitude, 2 sec ON, 1 sec OFF) in an ice bath to form a series of stable composite dispersions. Each dispersion was chemically reduced by taking $10 \mathrm{~mL}$ of composite dispersion and adding $10 \mathrm{~mL}$ of MilliQ water, $10 \mu \mathrm{L}$ of $35 \mathrm{wt} \%$ hydrazine and $70 \mu \mathrm{L}$ of $28 \mathrm{wt} \%$ ammonia solutions. The mixture was then shaken vigorously for 5 minutes and left for 1 hour at $95^{\circ} \mathrm{C}$ for reduction to take place. The final concentration of $\mathrm{rGO}$ was $0.25 \mathrm{mg} / \mathrm{mL}$ after reduction and the weight fraction of MWCNTs for each sample is given by the following labelling system throughout this manuscript. rGO-MWCNT 10 represents a rGO-MWCNT dispersion containing $10 \%$ wt MWCNTs or rGO-MWCNT 90 to represent a rGO-MWCNT dispersion containing $90 \mathrm{wt} \%$ MWCNTs and so on. Additionally, when the label rGO is used it signifies that no 
MWCNTs are present and similarly, when MWCNT is used it signifies that no rGO is present. The composite dispersions were characterised by X-ray Photoelectron Spectroscopy (XPS), optical microscopy (Leica optical microscope), scanning electron microscopy (SEM) (JEOL Cold Field Emission Gun Scanning Electron Microscope), zeta potential and particle size analysis (Zetasizer Nanoseries, Malvern Instruments). The dispersions were dried at $80^{\circ} \mathrm{C}$ under vacuum overnight before Fourier Transform - Infrared (FT-IR) analysis. An IRPrestige-21 Fourier transform infrared spectrophotometer (Shimadzu) was used to analyze the functional groups. $\mathrm{KBr}$ powder was used and the scanning was performed in the range of $4000-400 \mathrm{~cm}^{-1}$.

\subsection{Electrode fabrication and characterisation}

Glassy carbon electrodes (GCE) of $3 \mathrm{~mm}$ diameter were polished using a series of alumina powder slurries with various particle sizes, namely $1 \mu \mathrm{m}, 0.3 \mu \mathrm{m}, 0.1 \mu \mathrm{m}$ and $0.05 \mu \mathrm{m}$. The electrodes were then subjected to bath sonication in MilliQ water for 10 minutes, before being dried by a nitrogen

stream. $10 \mu \mathrm{L}$ of the above rGO-MWCNT dispersions was added to $10 \mu \mathrm{L}$ of purified GOx $(0.5$ $\mathrm{mg} / \mathrm{mL}$ in 50mM PBS, pH 7 - unless otherwise stated) and gently mixed by pipette before drop casting $5 \mu \mathrm{L}$ onto a clean GCE. These electrodes were placed in a refrigerator at $5^{\circ} \mathrm{C}$ and left until dry before a final layer of poly(ethyleneimine) (PEI) ( $5 \mu \mathrm{L}$ of $1 \mathrm{mg} / \mathrm{mL}$ in $0.5 \mathrm{M} \mathrm{NaCl}$ ) was drop cast to entrap the enzyme, and again allowed to dry at $5^{\circ} \mathrm{C}$

A series of electrodes were also prepared in a similar fashion for electrochemical impedance spectroscopy (EIS) analysis, however the rGO-MWCNT aqueous dispersions were drop cast without the GOx and PEI layers. Once dry, the electrodes were electrochemically tested in $10 \mathrm{mM}$ ferrocenemethanol (FcMeOH) in PBS (50 mM, pH 7) to characterise the charge transfer resistance of each rGO-MWCNT composition. EIS was performed at an oxidising potential for FcMeOH $(+0.28 \mathrm{~V}$ vs. Ag/AgCl reference electrode) in the frequency range 0.1 to $100000 \mathrm{~Hz}$ at an AC amplitude of 5 $\mathrm{mV}$. 
All enzyme electrochemistry tested with glucose in solution (henceforth referred to as the catalytic response) was performed at $37^{\circ} \mathrm{C}$ with a $\mathrm{PBS}(\mathrm{pH} 7,50 \mathrm{mM})$ supporting electrolyte under a blanket of argon gas. Initially the dissolved oxygen was removed from the electrolyte by bubbling argon through for at least $15 \mathrm{~min}$. Cyclic voltammetry was used to characterise the electrodes both with and without enzyme for 20 cycles at $50 \mathrm{mV} / \mathrm{s}$, which was sufficient to achieve stable electrochemistry before the sweep rate was dropped to $10 \mathrm{mV} / \mathrm{s}$ for steady state analysis.

\section{Results and Discussion}

\subsection{Dispersion and electrode properties}

MWCNTs were dispersed by sonication in an aqueous dispersion of GO prior to the reduction process. GO-MWCNT dispersions were characterized by X-ray photoelectron spectroscopy (XPS) before and after hydrazine reduction to quantify the ratio of carbon to oxygen containing groups in the final dispersion. It is evident in Figure 1(a) and (b) that there is a significant decrease in C-O content $(286 \mathrm{eV})$ relative to the $\mathrm{C}=\mathrm{C} / \mathrm{C}-\mathrm{C}$ peak $(284 \mathrm{eV})$ after chemical reduction of a $\mathrm{GO}$ dispersion [45][46]. This shows the extent of oxygen defects removed from the GO sheets that contribute to the number of $\mathrm{sp}^{2}$ bonded carbon sites, directly affecting the electrical resistivity of the material [47][48]. The peak at $288 \mathrm{eV}$ corresponds to $\mathrm{C}=\mathrm{O}$ groups, which are the remaining oxygen containing groups responsible for keeping the rGO dispersion electrostatically stable [7].
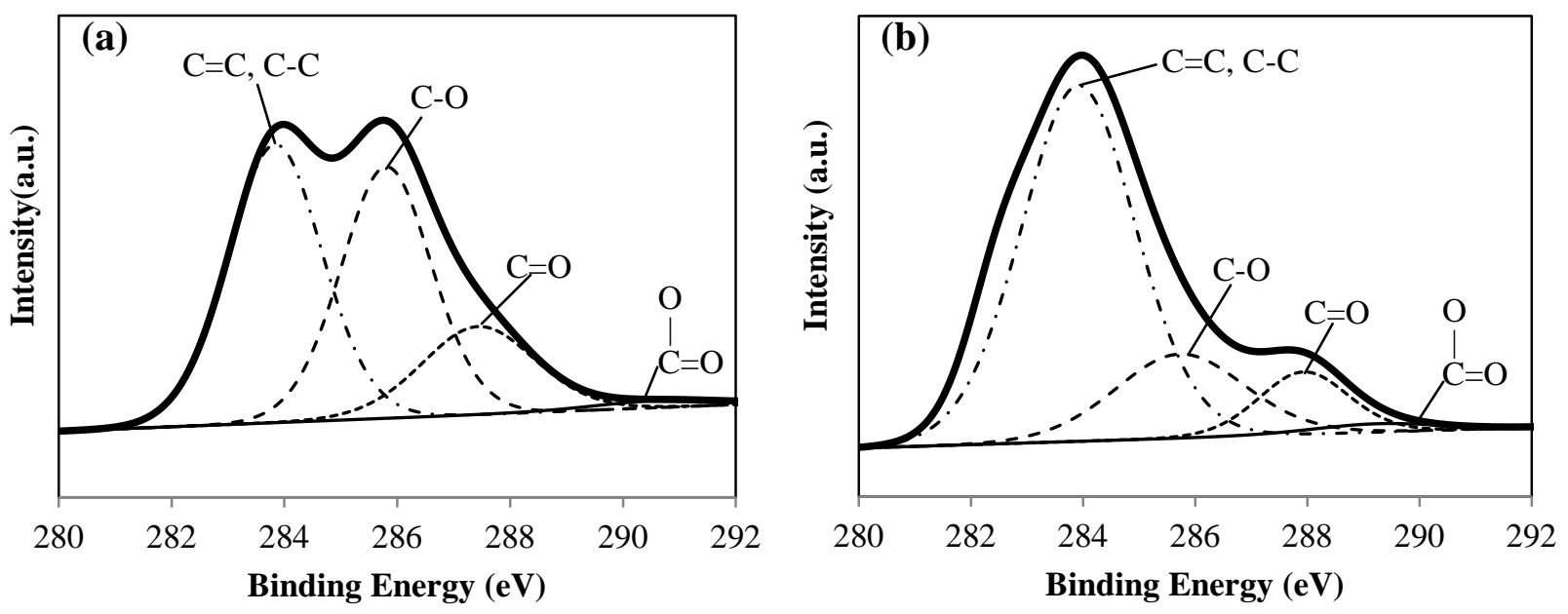
Figure 1 XPS data for GO dispersions before (a) and after (b) hydrazine reduction.

The same characterisation was performed on a GO-MWCNT 50 dispersion (data not shown), however, the carbon-carbon peak at $284 \mathrm{eV}$ dominated the spectra and the carbon to oxygen ratio was difficult to determine. Using XPS analysis it is inconclusive whether reduction of GO occurs in the presence of MWCNTs. FT-IR analysis (Figure 2) suggests that chemical reduction in fact does occur with the disappearance of the broad carboxylic acid band at $\sim 3100 \mathrm{~cm}^{-1}$ after reduction for samples with and without MWCNTs present, as well as the feature at $1724 \mathrm{~cm}^{-1}$ which corresponds to $\mathrm{C}=\mathrm{O}$ stretching vibration peak that also diminishes after reduction[49]. The disappearance of the oxygen containing group features indicates chemical reduction has occurred in the presence of MWCNTs.

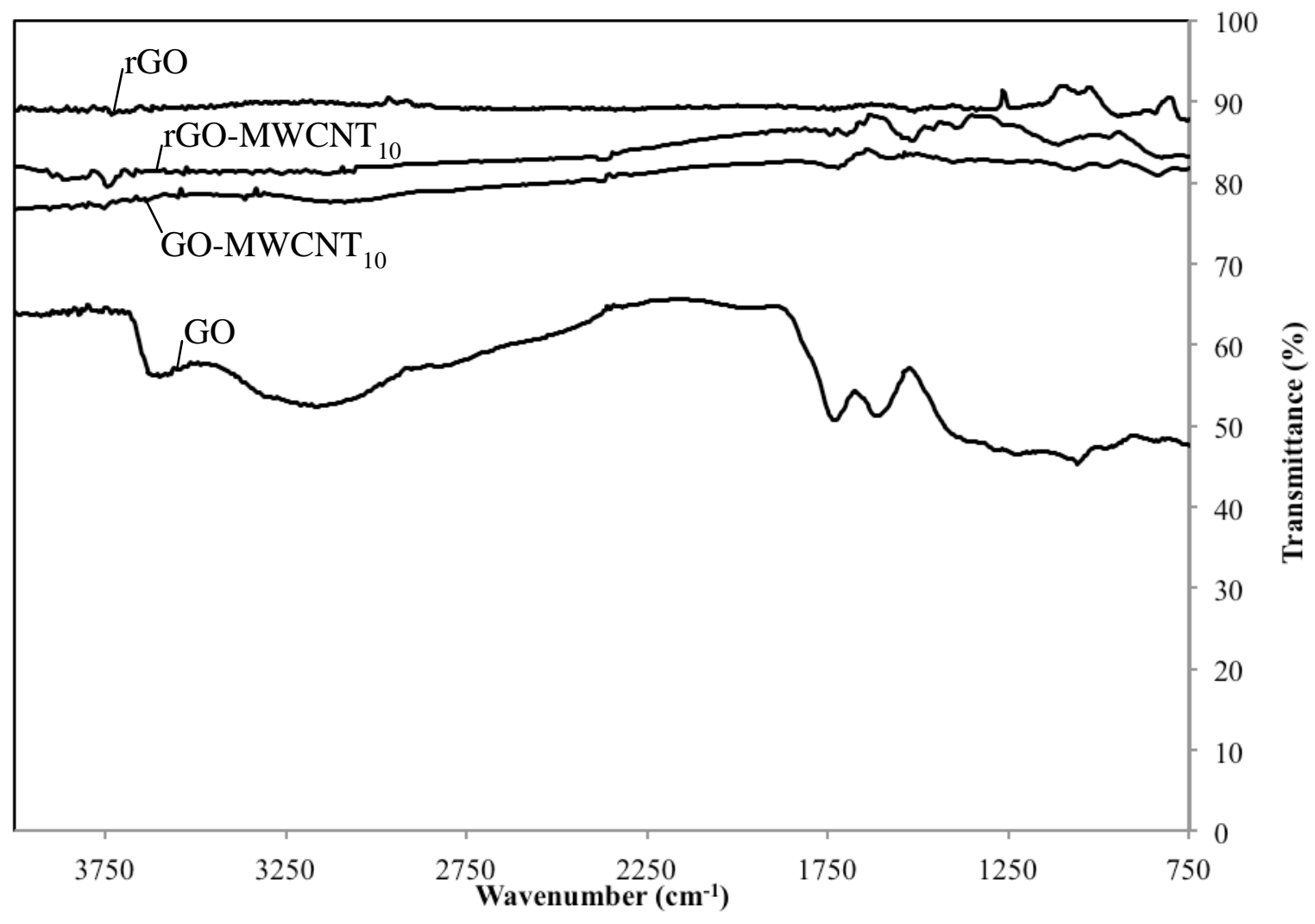

Figure 2 FT-IR spectra of GO, rGO, GO-MWCNT 10 and rGO-MWCNT 10 . 
It has been reported [7] that rGO has a higher conductivity than GO, and therefore electrochemical impedance spectroscopy (EIS) was used to investigate the electrical properties (namely the charge transfer resistance $-\mathrm{R}_{\mathrm{ct}}$ ) of films formed from GO-MWCNT dispersions before and after the chemical reduction treatment. A decrease in the $\mathrm{R}_{\mathrm{ct}}$ after chemical reduction of a film formed from a GO-MWCNT dispersion would indicate that the GO has been reduced to its more conducting rGO form. For the GO dispersions with $0 \mathrm{wt} \%$ MWCNTs, the $\mathrm{R}_{\mathrm{ct}}$ before and after chemical reduction was $166 \pm 31 \Omega$ and $108 \pm 2 \Omega$, respectively. This indicates that rGO is significantly more conducting than GO and supports XPS data suggesting reduction of GO has occurred. Figure 3 shows that for all weight \% MWCNTs the rGO samples exhibit a lower $\mathrm{R}_{\mathrm{ct}}$ compared to the same samples before reduction suggesting that reduction has occurred in the presence of MWCNTs and highlighting the fabrication of a conducting composite dispersion. There is a notable decrease in $\mathrm{R}_{\mathrm{ct}}$ before reduction (GO profile, Figure 3) as the amount of MWCNTs was increased from $0 \mathrm{wt} \%$ to $33 \mathrm{wt} \%$ due to the MWCNT's inherently high conductivity. There is no statistical difference for samples that have higher MWCNT contents than $33 \mathrm{wt} \%$ in this data set, suggesting the percolation threshold has been reached. 


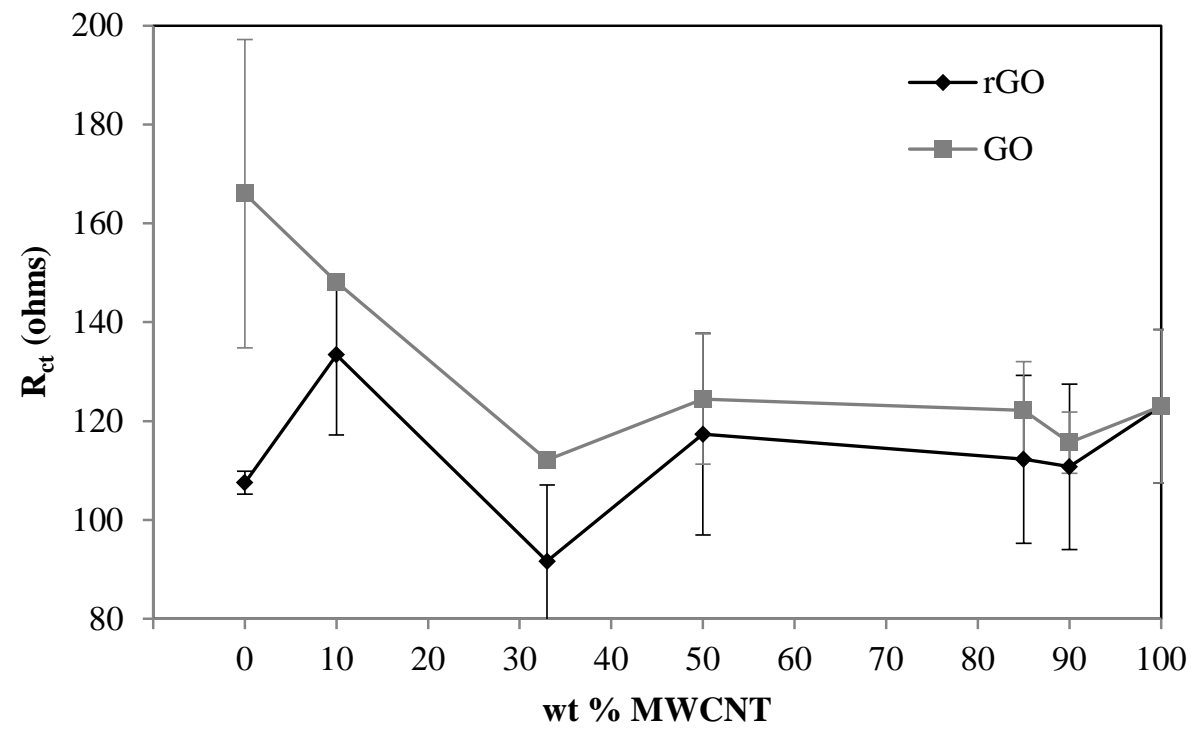

Figure 3 Charge transfer resistance of GO and rGO drop cast electrodes as a function of MWCNT loading.

The reduced dispersions were drop cast onto aluminium foil for SEM analysis with Figure 4 showing each rGO-MWCNT ratio. The rGO (Figure 4a) exhibits the characteristic wrinkle features [50] of drop cast graphene. As the MWCNT content is increased from rGO-MWCNT 10 (Figure 4b) through to rGO-MWCNT 90 (Figure 4e), the MWCNT features become dominant with some rGO wrinkles still visible. For MWCNT (Figure 4f) samples characteristic features of entangled MWCNTs are evident and it seems to be a less homogenous film compared to when rGO is present. The insets in Figure 4 are the optical micrographs of each of the respective dispersions used to form the films for SEM. The optical micrographs demonstrate the quality of each dispersion ratio and it is evident that rGO is very effective as a dispersing agent given that when no rGO is present (inset Figure 4f) MWCNT aggregation is obvious. It was also observed that the qualities of the dispersions before and after chemical reduction were identical thus highlighting the attractive nature of this rGO-MWCNT dispersion synthesis route. 

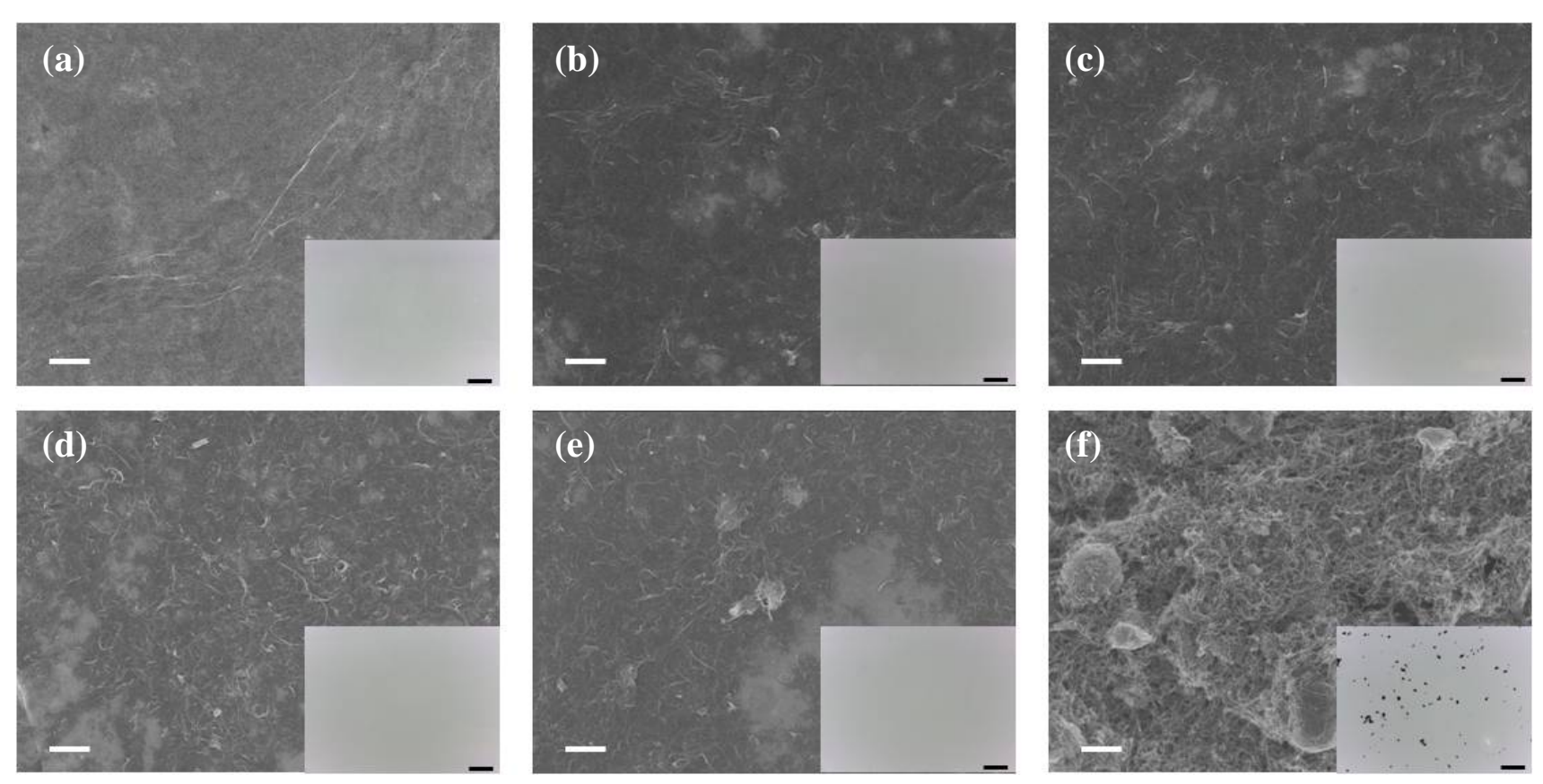

Figure 4 SEM micrographs of rGO-MWCNT composite dispersions at various weight \% MWCNTs drop cast on to Al foil. Inset: Optical images for each dispersion. White scale bars represent $1 \mu \mathrm{m}$ for SEM images and black scale bars represent $1 \mathrm{~mm}$ for optical images. (a) rGO, (b) rGO-MWCNT 10 , (c) rGO-MWCNT 33 , (d) rGO.MWCNT 85 , (e) rGO.MWCNT 90 and (f) MWCNT.

The dispersion properties were also characterised by SEM and optical microscopy after the addition of the glucose oxidase (GOx) enzyme to the dispersions (Figure 5). The images in Figure 5 show the films formed from rGO-MWCNT 10 with (a) and without (b) the addition of GOx and rGOMWCNT $_{90}$ with (c) and without (d) GOx addition. It is evident in both samples that rGO and MWCNTs form a homogenous film (Figure 5a and c) and when the enzyme is present the biological entity is incorporated into the homogenous film (Figure $5 \mathrm{~b}$ and $\mathrm{d}$ ). Carbon nanotube features are more predominant in the rGO-MWCNT 90 sample, as expected, and show thorough interaction between the carbon nanomaterials and the enzyme with MWCNTs protruding through the characteristic features of GOx. The optical micrographs (inset Figure 5) show that dispersion quality is not sacrificed when enzymes are present. These results give some indication that firstly, the 
enzyme does not destabilize the dispersion, and secondly, that there seems to be extensive physical interaction between the enzyme and the carbon materials which may facilitate DET.
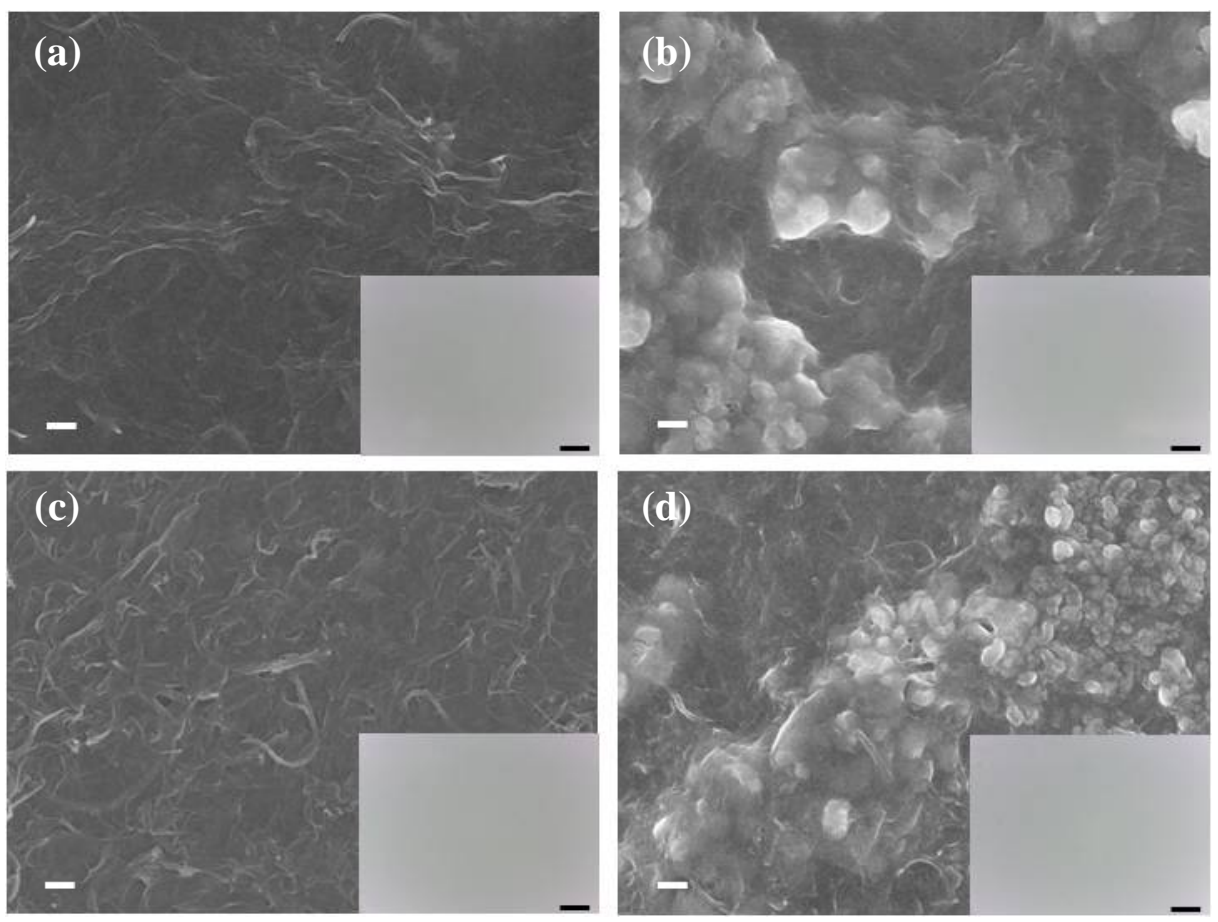

Figure 5 SEM micrographs of rGO-MWCNT and rGO-MWCNT-GOx composite dispersions drop cast on to Al foil. Inset: optical images for each dispersion. White scale bars represent $200 \mathrm{~nm}$ for SEM images and black scale bars represent $1 \mathrm{~mm}$ for optical images. (a) rGO-MWCNT 10 , (b) rGOMWCNT $_{10}-\mathrm{GOx}$, (c) rGO-MWCNT 90 and (d) rGO-MWCNT $90-G O x$.

Zeta potential and size analysis was carried out on rGO dispersions of various weight \% MWCNTs and each demonstrated similar and reproducible zeta potentials averaged at $-45.6 \mathrm{mV}$, all within an average standard deviation of $9.6 \mathrm{mV}$. Zeta potential values more negative than $-30 \mathrm{mV}$ are considered to represent sufficient electrostatic repulsion to ensure a stable dispersion [51]. There was no trend in zeta potential in relation to the amount of MWCNTs present, which indicates the entire 
rGO-MWCNT concentration range represents electrostatically stable systems. Particle size analysis gives more quantitative information given that through optical analysis the MWCNT sample was obviously not a stable dispersion (inset Figure 4f). The particle size distribution analysis showed good consistency for all samples from rGO through to rGO-MWCNT $_{90}$ which had average polydispersity index (PDI) values for day 1 and day 50 of $0.271 \pm 0.04$ and $0.324 \pm 0.06$, respectively (Table 1). These relatively large PDI values arise from the nature of the analysis which assumes spherical particles, with an ideal spherical, narrow monomodal system having a PDI $<0.1$. The dispersions, however, are composed of relatively flat sheets of rGO and cylindrical particles of MWCNTs so the following particle size data analysis is only relevant for comparison within this experiment. It is evident that there is a higher PDI value for the 50-day particle size analysis compared to day 1 which indicates there is an increase in particle size distribution and may indicate the onset of dispersion destabilisation, however, on analysis of the number average particle size there is no statistical difference between day 1 and day 50. There is no observable evidence of agglomeration after 50 days of standing and in conjunction with particle size analysis it suggests that the dispersions where rGO is present (i.e. rGO to rGO-MWCNT 90 ) are stable for over 50 days. The poor dispersion quality of the MWCNT sample described previously was confirmed through particle size analysis which showed a PDI value of 1.000 from day 1 . This result highlights the necessity of rGO to act as a dispersing agent for the highly insoluble MWCNTs.

Table 1 Nominal number average particle size and PDI data collected at day 1 and day 50 for the various compositions of rGO and MWCNTs

\begin{tabular}{|l|c|c|c|c|}
\cline { 2 - 5 } \multicolumn{1}{c|}{} & \multicolumn{2}{c|}{ Day 1 } & \multicolumn{2}{c|}{ Day 50 } \\
\hline Dispersion & Nominal Number & PDI & Nominal Number & PDI \\
& Average Particle size & & Average Particle size \\
& $(\mathbf{n m})$ & & $(\mathbf{n m})$ & 0.325 \\
\hline rGO & $339( \pm 172)$ & 0.257 & $385( \pm 220)$ & 0.283 \\
\hline rGO-MWCNT & $119( \pm 71)$ & 0.352 & $123( \pm 66)$ & \\
\hline
\end{tabular}




\begin{tabular}{|l|c|c|c|c|}
\hline rGO-MWCNT $_{33}$ & $133( \pm 68)$ & 0.263 & $170( \pm 105)$ & 0.382 \\
\hline rGO-MWCNT $_{50}$ & $152( \pm 78)$ & 0.265 & $201( \pm 120)$ & 0.358 \\
\hline rGO-MWCNT $_{85}$ & $168( \pm 86)$ & 0.262 & $170( \pm 80)$ & 0.219 \\
\hline rGO-MWCNT & & & $226( \pm 138)$ & 0.377 \\
\hline MWCNT & $166( \pm 79)$ & 0.228 & $3279( \pm 3279)$ & 1.000 \\
\hline
\end{tabular}

There is an interesting drop in nominal number average particle size between rGO and rGO$\mathrm{MWCNT}_{10}$ at both day 1 and day 50. It is unclear as to why the particle size is larger for rGO alone but may be attributed to the way the graphene sheets and the CNTs interact in solution. Some suggest the sheets can wrap around the CNTs [18] while Shin et al. suggest the CNTs are attached to the edges and surfaces of the graphene sheets [52]. When GOx was added to the rGO-MWCNT composite dispersions on day 1 there was no statistical variation in number average particle size between before and after enzyme addition, $180 \pm 80 \mathrm{~nm}$ and $164 \pm 31 \mathrm{~nm}$, respectively. This indicates that enzymes do not cause instabilities in the rGO- MWCNT dispersions.

\section{2 rGO-MWCNT-GOx Electrodes}

The aqueous nature of the rGO-MWCNT dispersions make them amenable to incorporation of enzymes for use in biological applications. Graphene [32] and carbon nanotubes [26] have been investigated separately for their ability to facilitate direct electron transfer (DET) in enzymatic systems. Here we test the validity of using a composite of these materials to achieve an enhanced enzymatic catalytic response. In order to investigate the ability of the composite electrodes to facilitate DET with immobilised GOx, the enzymes were mixed in the given composite dispersion and drop cast onto a polished GCE, immobilised with a thin layer of PEI and electrochemically tested in PBS $(\mathrm{pH} 7,50 \mathrm{mM})$ at $37^{\circ} \mathrm{C}$ under an argon blanket.

The $\mathrm{pH}$ of all the rGO-MWCNT dispersion was measured to be 10.0 and it was unknown if the GOx would remain in its active state in films formed from the basic dispersions. We have observed a 
significant decrease in the enzyme activity when moving from a $\mathrm{pH}$ of 7 to 10 (see Supporting Information). This decrease in activity is attributed to a change in the enzyme structure [53]. Cyclic voltammetry (Figure 6) performed on these rGO-MWCNT-GOx electrodes in PBS (pH 7.4) clearly show a pair of well defined redox peaks with anodic peak $\left(\mathrm{E}_{\mathrm{pa}}\right)$ and cathodic peak $\left(\mathrm{E}_{\mathrm{pc}}\right)$ at $-455 \mathrm{mV}$ and $-470 \mathrm{mV}$ respectively, corresponding to $15 \mathrm{mV}$ for $\Delta E_{\mathrm{p}}$ and a $\mathrm{E}^{\circ}$ of $-0.463 \mathrm{~V}$. The $\mathrm{E}^{\circ}$ value of GOx incorporated into the rGO-MWCNT electrode is in accordance with the typical characteristics of GOx electrochemistry in neutral $\mathrm{pH}$ solution [54]. This result demonstrates that despite GOx being incorporated into an rGO-MWCNT dispersion at pH 10 it still maintains its electroactivity in films formed from these dispersions. When GOx is drop cast directly onto GCE (i.e., no nanomaterial present) no peaks are evident (Figure 6d) which is to be expected as many publications have investigated the relationship between carbon nanomaterials and their capacity to facilitate DET compared to bare electrodes with no nanomaterials [27][55]. Figure 6 shows other cyclic voltammogram profiles for rGO, rGO-MWCNT 90 and MWCNT bioelectrode samples (Figure 6a, b \& c, respectively). A large capacitive response is observed for the rGO containing samples, attributed to graphene's high capacitance [46]. Its ability to communicate with immobilised enzymes is evident due to the oxidation and reduction peaks, which indicate the oxidation of GOx (FAD) to GOx $\left(\mathrm{FADH}_{2}\right)$ and its subsequent reduction (equation 2). Interestingly, the MWCNT sample shows a slightly more resistive response and less defined redox peaks, contrary to other literature where an obvious GOx redox response [56] on CNT drop cast films is evident. This may be the result of the unstable nature of the dispersion with MWCNTs preferring to agglomerate together rather than interact with the enzyme, however, it is still unclear.

The stability of the rGO-MWCNT 10 -GOx electrodes were characterised for a period of time under constant potential cycling at $10 \mathrm{mV} / \mathrm{s}$ in PBS (50 mM, pH7) between -0.7 to $-0.2 \mathrm{~V}$. It was evident that without the immobilizing PEI layer being present the electrode lost $100 \%$ of its DET signal after 1 hour, however, when a PEI layer is used to secure the components, the electrode retains $73 \%$ of its 
anodic peak height after 4 hours of cycling. This result highlights the importance of the PEI layer to keep the enzyme immobilised.

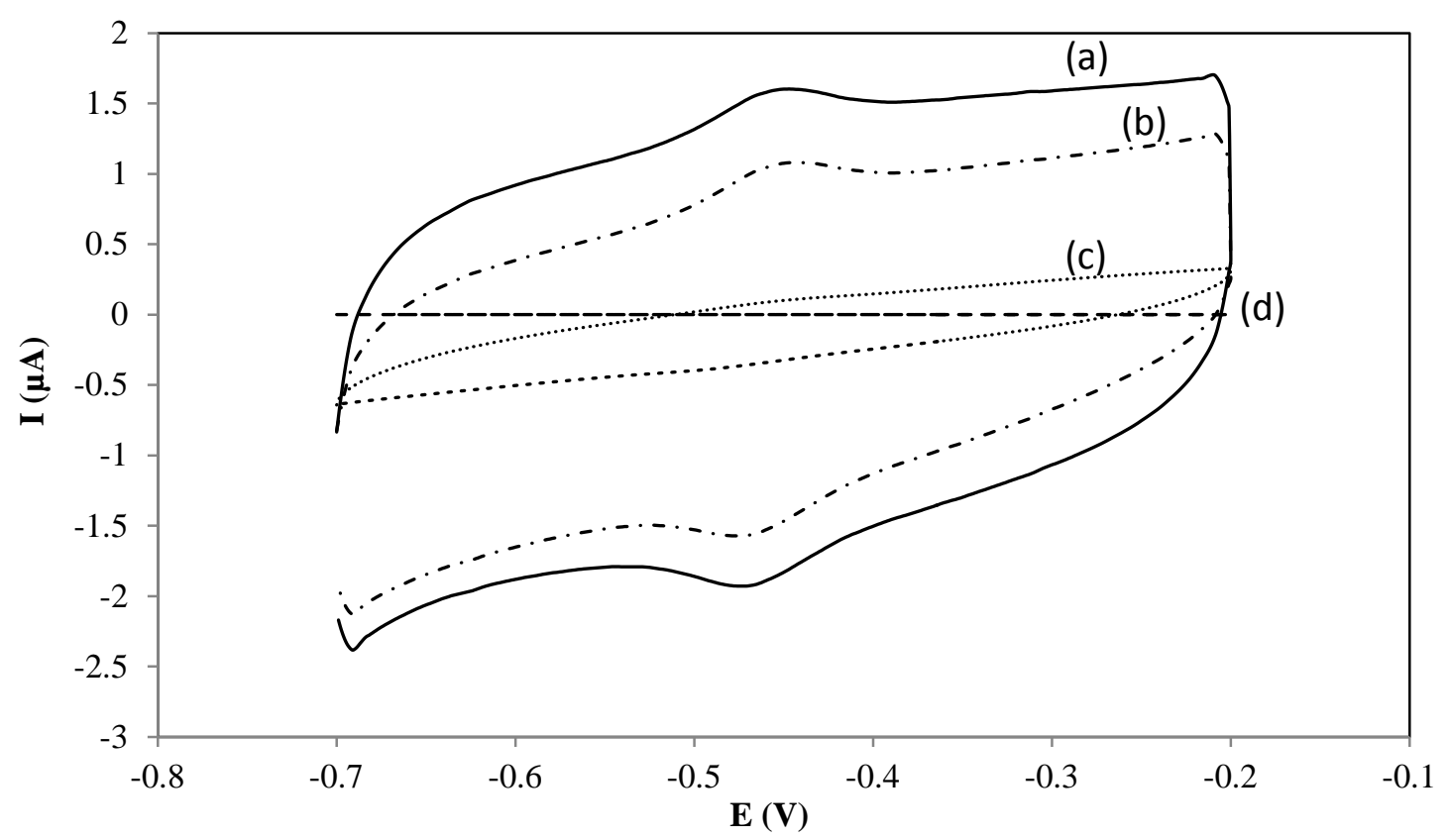

Figure 6 Cyclic voltammograms of electrodes (a) rGO-GOx (b) rGO-MWCNT $90-G O x$, (c) MWCNT-GOx and (d) GOx immobilised with PEI with no carbon nanomaterials present, in PBS at $37^{\circ} \mathrm{C}$ under argon at a scan rate of $10 \mathrm{mV} / \mathrm{s}$ (vs. $\mathrm{Ag} / \mathrm{AgCl}$ ).

Enzymatic catalytic current can be analysed through the addition of glucose to the PBS solution and monitoring the change in anodic peak $\left(\mathrm{I}_{\mathrm{pa}}\right)$ height (after subtraction of the appropriate capacitive background current) (Figure 7a). This data provides information on the effectiveness of each composite electrode to facilitate DET, with larger changes indicating enhanced electron transfer efficiency from the FAD redox centre of the GOx to the composite electrode material. The catalytic current is plotted for each composite electrode (Figure 7b) with significant variation associated with the higher weight \% MWCNT samples and more reproducible catalytic currents recorded for low weight \% MWCNT. The variable response with increasing MWCNT content can be attributed to the 
increase in film heterogeneity, which may be detrimental to the direct wiring of GOx. Catalytic analysis was not possible for the MWCNT sample since the $\mathrm{I}_{\mathrm{pa}}$ was undetectable.
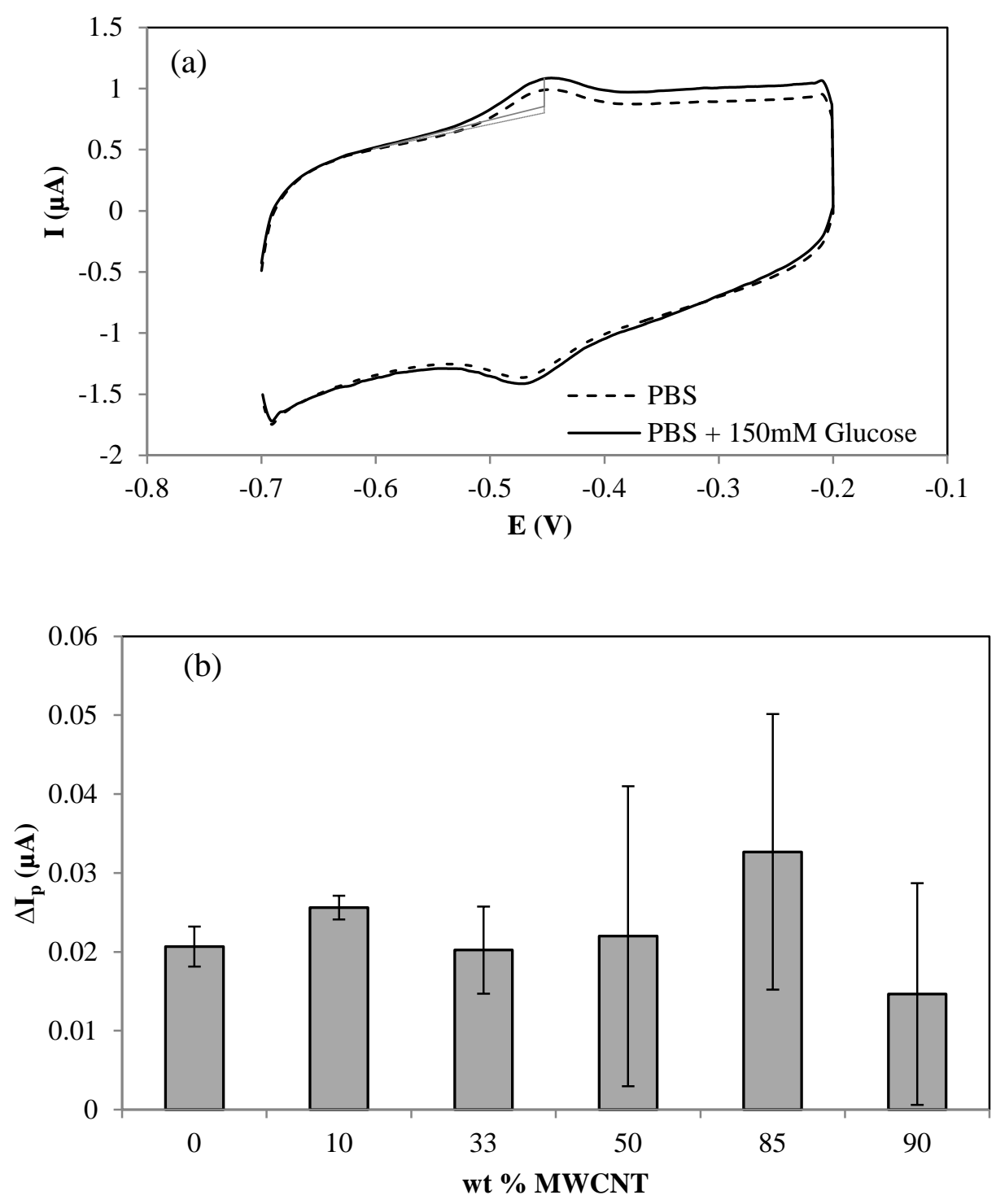

Figure 7 (a) An example of an increase in $\mathrm{I}_{\mathrm{pa}}$ in response to $150 \mathrm{mM}$ glucose in solution for an rGOMWCNT $_{90}-\mathrm{GOx}$ sample vs. Ag/AgCl and (b) the catalytic current as a function of wt\% MWCNTs. 
The observed catalytic responses presented in Figure 7 indicate that the glucose can access the enzyme and that this enzyme is electrically wired to the electrode. These values can be directly compared to analyse the efficiency of each rGO-MWCNT ratio to facilitate DET. In this case the largest reproducible catalytic response came from the rGO-MWCNT 10 composition. The rGOMWCNT $_{10}$ system was subsequently used to determine the optimal GOx loading in terms of current generated per amount of GOx used $(\mu \mathrm{A} / \mu \mathrm{g})$. The average specific catalytic current for our best performing electrode (rGO-MWCNT $10-\mathrm{GOx}$ ) composite, with an optimised enzyme loading of 0.002:1 with respect to rGO (i.e., GOx: rGO), was $72 \mu \mathrm{A} / \mu$ g GOx at 50mV/s. Other literature values for DET systems to date, using electrodes prepared using the same drop cast method, report 0.5 $\mu \mathrm{A} / \mu \mathrm{g}$ of GOx for a MWCNT/CTAB/GOx/Nafion composite [55], $0.12 \mu \mathrm{A} / \mu \mathrm{g}$ GOx for a Graphene Oxide/Chitosan/GOx electrode [57] and $0.06 \mu \mathrm{A} / \mu \mathrm{g}$ GOx for a mesoporous carbon/nafion/GOx system [58], at the same scan rate. This equates to a 144-fold increase in efficiency for the electrodes prepared in this manuscript compared to the closest literature value. The rGOMWCNT $_{90}-\mathrm{GOx}$ and rGO-GOx samples measured 65 and $64 \mu \mathrm{A} / \mu \mathrm{g}$ of GOx, respectively, also demonstrating an enhanced specific catalytic performance. The significant improvement in specific catalytic current for the rGO-MWCNT composite electrodes compared to other literature values can be attributed to the higher activity achieved through enzyme purification and the ability of the composite to facilitate efficient direct electron transfer.

\section{Conclusions:}

We have optimised the process of fabricating stable solutions of highly conducting rGO and MWCNTs in an aqueous system without the need for additional stabilising agents. Dispersion quality was studied before and after the addition of the enzyme glucose oxidase, and it was determined through SEM, optical microscopy, zeta potential and size analysis that the dispersions remain stable for all rGO-MWCNT composites with MWCNT content between 10 and 90 wt\%. 
MWCNTs alone did not form stable dispersions under any conditions due to their extremely low solubility in water. This result highlights how effectively rGO acts as a dispersing agent for the otherwise insoluble MWCNTs. The optimal bioelectrodes were determined to be rGO-MWCNT $10^{-}$ GOx which produced the largest reproducible catalytic response for the compositions tested, and upon optimisation of enzyme loading proved to out-perform other literature values by a factor of 144 for specific catalytic current generated. The exceptional specific performance of the electrodes is attributed to the efficiency of the composite materials to facilitate direct electron transfer and the high specific activity of the purified enzyme. The ability to fabricate aqueous dispersions of highly conducting materials in conjunction with biological entities, like enzymes, provides opportunities for implementing other solution processable techniques to form a range of bioelectrodes.

\section{Acknowledgements:}

The authors wish to thank the Australian Research Council (ARC) for their financial support. G. G. Wallace wishes to thank the ARC for his Laureate Fellowship and S. E. Moulton wishes to thank the ARC for his Queen Elizabeth II Fellowship. The authors acknowledge funding support from the Australian Nanofabrication Facility, the assistance of Dr Tony Romeo for SEM images and the University of Montpellier 2 for their support of Joffrey Champavert during his time in Australia.

\section{Figure Captions:}

Figure 8 XPS data for GO dispersions before (a) and after (b) hydrazine reduction.

Figure 9 FT-IR spectra of GO, rGO, GO-MWCNT 10 and rGO-MWCNT $_{10}$.

Figure 10 Charge transfer resistance of GO and rGO drop cast electrodes as a function of MWCNT loading.

Figure 11 SEM micrographs of rGO-MWCNT composite dispersions at various weight \% MWCNTs drop cast on to Al foil. Inset: Optical images for each dispersion. White scale bars 
represent $1 \mu \mathrm{m}$ for SEM images and black scale bars represent $1 \mathrm{~mm}$ for optical images. (a) rGO, (b) rGO-MWCNT $_{10}$, (c) rGO-MWCNT 33 , (d) rGO.MWCNT 85 , (e) rGO.MWCNT90 and (f) MWCNT.

Figure 12 SEM micrographs of rGO-MWCNT and rGO-MWCNT-GOx composite dispersions drop cast on to Al foil. Inset: optical images for each dispersion. White scale bars represent $200 \mathrm{~nm}$ for SEM images and black scale bars represent $1 \mathrm{~mm}$ for optical images. (a) rGO-MWCNT 10 , (b) rGOMWCNT $_{10}-\mathrm{GOx}$, (c) rGO-MWCNT 90 and (d) rGO-MWCNT $90-\mathrm{GOx}$.

Table 2 Nominal number average particle size and PDI data collected at day 1 and day 50 for the various compositions of rGO and MWCNTs

Figure 13 Cyclic voltammograms of electrodes (a) rGO-GOx (b) rGO-MWCNT $_{90}-\mathrm{GOx}$, (c) MWCNT-GOx and (d) GOx immobilised with PEI with no carbon nanomaterials present, in PBS at $37^{\circ} \mathrm{C}$ under argon at a scan rate of $10 \mathrm{mV} / \mathrm{s}$ (vs. $\mathrm{Ag} / \mathrm{AgCl}$ ).

Figure 14 (a) An example of an increase in $I_{p a}$ in response to $150 \mathrm{mM}$ glucose in solution for an rGO-MWCNT ${ }_{90}-\mathrm{GOx}$ sample vs. Ag/AgCl and (b) the catalytic current as a function of wt\% MWCNTs.

\section{References:}

[1] Dalton A, Collins S, Muñoz E, Razal J, Ebron V, Ferraris J, Coleman J, Kim B, and Baughman R. Super-tough carbon-nanotube fibres. Nature 2003;423:703.

[2] Meitl M, Zhou Y, Gaur A, Jeon S, Usrey M, Strano M, and Rogers J. Solution Casting and Transfer Printing Single-Walled Carbon Nanotube Films. Nano Letters 2004;4(9):1643-1647.

[3] Tong X, Zheng J, Lu Y, Zhang Z, and Cheng H. Swelling and mechanical behaviors of carbon nanotube/poly(vinyl alcohol) hybrid hydrogels. Materials Letters 2007;61:1704-1706.

[4] Chen J, Hamon M, Hu H, Chen Y, Rao A, Eklund P and Haddon R. Solution Properties of Single-Walled Carbon Nanotubes. Science 1998;282:95-98.

[5] Rastogi R, Kaushal R, Tripathi S, Sharma A, Kaur I and Bharadwaj L. Comparative study of carbon nanotube dispersion using surfactants. Journal of colloid and interface science 2008;328(2):421-8. 
[6] Mattos C and Ringe D. Proteins in organic solvents. Current opinion in structural biology 2001;11(6):761-4.

[7] Li D, Müller M, Gilje S, Kaner R, and Wallace G. Processable aqueous dispersions of graphene nanosheets. Nature nanotechnology 2008;3(2):101-5.

[8] Kim Y and Min D. Durable large-area thin films of graphene/carbon nanotube double layers as a transparent electrode. Langmuir : the ACS journal of surfaces and colloids 2009;25(19):11302-6.

[9] Fan Z, Yan J, Zhi L, Zhang Q, Wei T, Feng J, Zhang M, Qian W, and Wei F. A threedimensional carbon nanotube/graphene sandwich and its application as electrode in supercapacitors. Advanced materials 2010;22(33):3723-8.

[10] Dreyer D, Park S, Bielawski C, and Ruoff R. The chemistry of graphene oxide. Chemical Society reviews 2010;39(1):228-40.

[11] Li Y, Zhou W, Wang H, Xie L, Liang Y, Wei F, Idrobo J, Pennycook S, and Dai H. An oxygen reduction electrocatalyst based on carbon nanotube-graphene complexes. Nature nanotechnology 2012;7:394-400.

[12] Tung V, Chen L, Allen M, Wassei J, Nelson K, Kaner R, and Yang Y. Low-temperature solution processing of graphene-carbon nanotube hybrid materials for high-performance transparent conductors. Nano letters 2009;9(5):1949-55.

[13] Zhang D, Yan T, Shi L, Peng Z, Wen X, and Zhang J. Enhanced capacitive deionization performance of graphene/carbon nanotube composites. Journal of Materials Chemistry 2012;22(29):14696-14704.

[14] Cai D, Song M, and Xu C. Highly Conductive Carbon-Nanotube/Graphite-Oxide Hybrid Films. Advanced Materials 2008;20(9):1706-1709.

[15] Artyukh A, Chernozatonskii L, and Sorokin P. Mechanical and electronic properties of carbon nanotube-graphene compounds. Physica Status Solidi (B) 2009;247(11-12):2927-2930.

[16] González J, Guinea F, and Herrero J. Propagating, evanescent, and localized states in carbon nanotube-graphene junctions. Physical Review B 2009;79(16):1-10.

[17] Sheka E and Chernozatonskii L. Graphene-Carbon Nanotube Composites. Computational and Theoretical Nanoscience 2010;7:1814-1824.

[18] Chen S, Yeoh W, Liu Q, and Wang G. Chemical-free synthesis of graphene-carbon nanotube hybrid materials for reversible lithium storage in lithium-ion batteries. Carbon 2012;50(12):4557-4565.

[19] Yang W, Choi H, Choi S, Jeon M, and Lee S. Carbon nanotube-graphene composite for ionic polymer actuators. Smart Materials and Structures 2012;21(5):1-7. 
[20] Yu A, Ramesh P, Sun X, Bekyarova E, Itkis M, and Haddon R. Enhanced Thermal Conductivity in a Hybrid Graphite Nanoplatelet - Carbon Nanotube Filler for Epoxy Composites. Advanced Materials 2008;20(24):4740-4744.

[21] Biswas C and Lee Y. Graphene Versus Carbon Nanotubes in Electronic Devices. Advanced Functional Materials 2011;21(20):3806-3826.

[22] Qiu L, Yang X, Gou X, Yang W, Ma Z, Wallace G, and Li D. Dispersing carbon nanotubes with graphene oxide in water and synergistic effects between graphene derivatives. Chemistry: A European Journal 2010;16(35):10653-8.

[23] Hu F, Chen S, Wang C, Yuan R, Yuan D, and Wang C. Study on the application of reduced graphene oxide and multiwall carbon nanotubes hybrid materials for simultaneous determination of catechol, hydroquinone, p-cresol and nitrite. Analytica chimica acta 2012;724(2):40-6.

[24] Tung V, Chen L, Allen M, Wassei J, Nelson K, Kaner R, and Yang Y. Low-Temperature Solution Processing of Graphene-Carbon Nanotube Hybrid Materials for High-Performance Transparent Conductors. Nano Letters 2009;9(5):1949-1955.

[25] Delvaux M and Demoustier-Champagne S. Immobilisation of glucose oxidase within metallic nanotubes arrays for application to enzyme biosensors. Biosensors \& bioelectronics 2003;18(7):943-51.

[26] Liu Y, Wang M, Zhao F, Xu Z, and Dong S. The direct electron transfer of glucose oxidase and glucose biosensor based on carbon nanotubes/chitosan matrix. Biosensors \& bioelectronics 2005;21(6):984-8.

[27] Zhao H, Sun J, Song J, and Yang Q. Direct electron transfer and conformational change of glucose oxidase on carbon nanotube-based electrodes. Carbon 2010;48(5):1508-1514.

[28] Zhao X, Jia H, Kim J, and Wang P. Kinetic Limitations of a Bioelectrochemical Electrode Using Carbon Nanotube-Attached Glucose Oxidase for Biofuel Cells. Biotechnology and Bioengineering 2009;104(6):1068-1074.

[29] Ivnitski D, Branch B, Atanassov P, and Apblett C. Glucose oxidase anode for biofuel cell based on direct electron transfer. Electrochemistry Communications 2006;8:1204-1210.

[30] Davis F and Higson P. Biofuel cells - Recent advances and applications. Biosensors and Bioelectronics 2007;22:1224-1235.

[31] Kang X, Wang J, Wu H, Aksay I, Liu J, and Lin Y. Glucose oxidase-graphene-chitosan modified electrode for direct electrochemistry and glucose sensing. Biosensors \& bioelectronics 2009;25(4):901-5.

[32] Wu P, Shao Q, Hu Y, Jin J, Yin Y, Zhang H, and Cai C. Direct electrochemistry of glucose oxidase assembled on graphene and application to glucose detection. Electrochimica Acta 2010;55(28):8606-8614. 
[33] Wang S, Zhang Q, Wang R, and Yoon S. A novel multi-walled carbon nanotube-based biosensor for glucose detection. Biochemical and Biophysical Research Communications 2003;311:572-576.

[34] Nien P, Wang J, Chen P, Chen L, and Ho K. Encapsulating benzoquinone and glucose oxidase with a PEDOT film: Application to oxygen-independent glucose sensors and glucose / O 2 biofuel cells. Bioresource Technology 2010;101:5480-5486.

[35] Salimi A and Noorbakhsh A. Layer by layer assembly of glucose oxidase and thiourea onto glassy carbon electrode: Fabrication of glucose biosensor. Electrochimica Acta 2011;56(17):6097-6105.

[36] Green R, Lovell N, Wallace G, and Poole-Warren L. Conducting polymers for neural interfaces: challenges in developing an effective long-term implant. Biomaterials 2008;29(2425):3393-9.

[37] Ranck J. Which elements are excited in electrical stimulation of mammalian central nervous system: a review. Brain Research 1975;98:417-440.

[38] Razal J, Kita M, Quigley A, Kennedy E, Moulton S, Kapsa R, Clark G, and Wallace G. WetSpun Biodegradable Fibers on Conducting Platforms: Novel Architectures for Muscle Regeneration. Advanced Functional Materials 2009;19(21):3381-3388.

[39] Nuzzo R. The future of electronics manufacturing is revealed in the fine print. Proceedings of the National Academy of Sciences of the United States of America 2001;98(9):4827-9.

[40] Hummers W and Offeman R. Preparation of Graphitic Oxide. 1958:1339.

[41] Kovtyukhova N, Ollivier P, Martin B, Malloukn T, Chizhik S, Buzaneva E, and Gorchinskiy A. Layer-by-Layer Assembly of Ultrathin Composite Films from Micron-Sized Graphite Oxide Sheets and Polycations. Chemistry of Materials 1999;11(3):771-778.

[42] Gao F, Courjean O, and Mano N. An improved glucose / O 2 membrane-less biofuel cell through glucose oxidase purification. Biosensors and Bioelectronics 2009;25:356-361.

[43] Bateman R and Evans J. Using the Glucose Oxidase / Peroxidase System in Enzyme Kinetics. Journal of Chemical Education 1995;72:240-241.

[44] Wilson R. Glucose oxidase : an ideal enzyme. Biosensors \& Bioelectronics 1992;7:165-185.

[45] De Silva K, Gambhir S, Wang X, Xu X, Li W, Officer D, Wexler D, Wallace G, and Dou S. The effect of reduced graphene oxide addition on the superconductivity of MgB2. Journal of Materials Chemistry 2012;22(28):13941-13946.

[46] Sheng K, Xu Y, Li C, and Shi G. High-performance self-assembled graphene hydrogels prepared by chemical reduction of graphene oxide. New Carbon Materials 2011;26(1):9-15.

[47] Mattevi C, Eda G, Agnoli S, Miller S, Mkhoyan K, Celik O, Mastrogiovanni D, Granozzi G, Garfunkel E, and Chhowalla M. Evolution of Electrical, Chemical, and Structural Properties 
of Transparent and Conducting Chemically Derived Graphene Thin Films. Advanced Functional Materials 2009;19(16):2577-2583.

[48] Robinson J, Perkins F, Snow E, Wei Z, and Sheehan P. Reduced graphene oxide molecular sensors. Nano letters 2008;8(10):3137-40.

[49] Zhang J, Yang H, Shen G, Cheng P, Zhang J, and Guo S. Reduction of graphene oxide via Lascorbic acid. Chemical Communications 2010;46(7):1112-4.

[50] Zhou X, Lu G, Qi X, Wu S, Li H, Boey F, and Zhang H. A Method for Fabrication of Graphene Oxide Nanoribbons from Graphene Oxide Wrinkles. Journal of Physical Chemistry C 2009;113(44):19119-19122.

[51] Everett D. Basic Principles of Colloid Science. London: Royal Society of Chemistry 1988.

[52] Shin M, Lee B, Kim S, Lee J, Spinks G, Gambhir S, Wallace G, Kozlov M, Baughman R, and Kim S. Synergistic toughening of composite fibres by self-alignment of reduced graphene oxide and carbon nanotubes. Nature communications 2012;3:650.

[53] Nelson D and Cox M. Lehninger Principles of Biochemistry (Fourth Edition). 2005:190-233.

[54] Bao S, Li C, Zang J, Cui X, Qiao Y, and Guo J. New Nanostructured TiO2 for Direct Electrochemistry and Glucose Sensor Applications. Advanced Functional Materials 2008;18(4):591-599.

[55] Cai C and Chen J. Direct electron transfer of glucose oxidase promoted by carbon nanotubes. Analytical Biochemistry 2004;332:75-83.

[56] Deng S, Jian G, Lei J, Hu Z, and Ju H. A glucose biosensor based on direct electrochemistry of glucose oxidase immobilized on nitrogen-doped carbon nanotubes. Biosensors \& bioelectronics 2009;25(2):373-7.

[57] Kang X, Wang J, Wu H, Aksay I, Liu J, and Lin Y. Glucose Oxidase - graphene - chitosan modified electrode for direct electrochemistry and glucose sensing. Biosensors and Bioelectronics 2009;25: pp. 901-905.

[58] Wang K, Yang H, Zhu L, Ma Z, Xing S, Lv Q, Liao J, Liu C, and Xing W. Direct electron transfer and electrocatalysis of glucose oxidase immobilized on glassy carbon electrode modified with Nafion and mesoporous carbon FDU-15. Electrochimica Acta 2009;54:46264630. 